AperTO - Archivio Istituzionale Open Access dell'Università di Torino

\title{
Anticoagulants used in plasma collection affect adipokine multiplexed measurements
}

\section{This is the author's manuscript}

Original Citation:

\section{Availability:}

This version is available http://hdl.handle.net/2318/1561914

since 2020-02-28T15:25:49Z

Published version:

DOI:10.1016/j.cyto.2016.03.001

Terms of use:

Open Access

Anyone can freely access the full text of works made available as "Open Access". Works made available under a Creative Commons license can be used according to the terms and conditions of said license. Use of all other works requires consent of the right holder (author or publisher) if not exempted from copyright protection by the applicable law. 
1 Anticoagulants used in plasma collection affect adipokine multiplexed 2 measurements

3

4 Alessandra Allione ${ }^{a, b,{ }^{*},}$ Cornelia Di Gaetano ${ }^{a, b}$, Nadia Danic, Davide Barberio ${ }^{c}$, Sabina

5 Sierid, Vittorio Kroghd, Giuseppe Matullo

6

7 a Human Genetics Foundation- HuGeF, Via Nizza 52, 10126 Turin, Italy

8 b Department of Medical Sciences, University of Turin, Via Santena 19, 10100

9 Turin, Italy

10 c Bioclarma Srl, Via Nizza 52, 10126 Turin, Italy

11 d IRCCS Foundation, Istituto Nazionale dei Tumori, Via Venezian, 1, 20133 Milan,

12 Italy

13

$14 *$ Corresponding author:

15 Human Genetics Foundation - HuGeF, Via Nizza 52, 10126 Turin, Italy

16 E-mail: alessandra.allione@hugef-torino.org

17

18 
19 Abstract

20 Obesity is an important health problem worldwide. Adipose tissue acts as an

21 endocrine organ that secretes various bioactive substances, called adipokines,

22 including pro-inflammatory biomarkers such as TNF- $\alpha$, IL-6, leptin and C-reactive

23 protein (CRP) and anti-inflammatory molecules such as adiponectin. The

24 deregulated production of adipokines in obesity is linked to the pathogenesis of

25 various disease processes and monitoring their variation is critical to understand

26 metabolic diseases.

27 The aim of this study was to determine the plasma concentration of adipokines in

28 healthy subjects by multiplexed measurements and the effect of anticoagulants on

29 their levels.

30 Plasma samples from 10 healthy donors were collected in two different

31 anticoagulants (sodium citrate or heparin).

32 All markers, excluding TNF- $\alpha$, showed significantly higher concentrations in

33 heparinized compared to citrate plasma. However, levels of adipokines in different

34 plasma samples highly correlated for most of these markers.

35 We reported that different anticoagulants used in the preparation of the plasma

36 samples affected the measurements of some adipokines. The importance of the

37 present results in epidemiology is relevant when comparing different studies in

38 which blood samples were collected with different anticoagulants.

40 Keywords: adipokines, anticoagulants, cytokines, microbead assay, plasma 


\section{Introduction}

42 Adipokines are defined as cell signaling mediators secreted by the adipose tissue. 43 They have both pro-inflammatory and anti-inflammatory activities, and the 44 imbalance between the different factors secreted by adipose tissue contributes to metabolic dysfunction. [1]. When adipocyte dysfunction is developed as a result of adipose tissue expansion, the deregulation of adipokine levels can produce several

47 effects on inflammatory responses, thereby contributing to the initiation and 48 progression of obesity-induced metabolic and cardiovascular complications [1]. 49 Therefore, further elucidation of the functions and mechanisms of key adipokines 50 will lead to a better understanding of the pathogenesis of obesity-linked disorders. 51 Adipokines are involved in the regulation of metabolism and insulin sensitivity. 52 Moreover, inflammation and blood concentrations of various adipokines are associated with obesity, metabolic and cardiovascular diseases. It is, therefore,

54 emerging that serum adipokine levels may serve as biomarkers of obesity-related 55 illnesses [2].

56 Recent papers demonstrated the effect of anticoagulants on multiplexed 57 measurement of cytokines/chemokines in healthy subjects [3-7], highlighting the 58 importance of sample preparation in biomarkers measurements in plasma. The 59 Luminex multiplex platform system is a highly efficient fluorescent (or magnetic) bead-based capture/detection sandwich immunoassay that allows for

61 measurements of multiple analytes simultaneously in a single reaction with small 62 sample volumes [8, 9]. This technology can measure up to 100 different analytes 63 using as little as $50 \mu \mathrm{l}$ sample volume, making it an assay very useful in clinical

64 trials or epidemiology studies, especially when volumes are limited. 
65 The aim of this study was to evaluate the effect of two different anticoagulants

66 (lithium heparin (LiEP) and sodium citrate (NaCitr)) on the levels of adipokines in

67 plasma collected from 10 healthy subjects. Multiplex microbead immunoassay was

68 performed for measuring IL-6, TNF- $\alpha$ and leptin while adiponectin and C-reactive

69 protein (CRP) were measured as a single measurement.

70

71 2. Materials and Methods

\section{$72 \quad 2.1$ Samples}

73 Samples were obtained concurrently from 10 healthy donors. Twenty milliliters of 74 peripheral venous blood were drawn into vacutainer tubes containing two 75 different anticoagulants (LiEp or NaCitr). Samples were centrifuged immediately 76 after the blood withdrawal, and plasma was analysed within 1 hour. Cells were 77 removed from plasma by centrifugation for 15 minutes at $2,000 \mathrm{x} \mathrm{g}$ at room 78 temperature. Written informed consent was achieved from all subjects. The study 79 protocol was approved by the ethics committee of the Fondazione IRCCS (Istituto 80 per la Ricerca e la Cura del Cancro, National Institute for Research and Treatment of Cancer) Istituto Nazionale dei Tumori (Milan, Italy).

82 Aliquots of the samples were stored at $-80^{\circ} \mathrm{C}$ and analysed ten days later.

83

\section{$84 \quad 2.2$ Experimental measurements}

85 The adipokine analysis was performed with the Luminex technology, which 86 combines the principle of a sandwich immunoassay with fluorescent bead-based 87 technology. In this way, individual and multiplex analysis of different analytes in a 88 single microwell plate are allowed [10]. Capture antibodies directed against the 
89 biomarker of interest are covalently coupled to fluorescently dyed magnetic 90 microspheres, each with a different color code or spectral address to allow 91 discrimination of individual tests within a multiplex suspension. Coupled beads 92 react with the sample containing the analyte of interest. After a series of washes to 93 remove unbound protein, a biotinylated detection antibody is added to create a 94 sandwich complex. The final detection complex is formed by the addition of 95 streptavidin-phycoerythrin (SA-PE) conjugate. Phycoerythrin serves as a 96 fluorescent indicator.

97 The assay for human IL-6, TNF- $\alpha$, leptin, adiponectin (Bio-Rad Laboratories, 98 Hercules California, USA) and CRP (Merk Millipore, Darmstadt, Germany) was 99 carried out on fresh plasma samples using 96-well microplates accordingly to the 100 recommendations of manufacturers. The contents of each well were moved into 101 the Bio-Plex 100 System array reader (Bio-Rad Laboratories Bio-Rad Laboratories, 102 Hercules California, USA), which identifies and quantifies each specific reaction 103 based on bead color and fluorescent signal intensity. The data were finally 104 processed using Bio-Plex Manager software (version 6.1) using five-parametric 105 curve fitting and converted in $\mathrm{pg} / \mathrm{ml}$.

106

\section{$107 \quad 2.3$ Statistical analyses}

108 All results are showed as mean \pm standard deviation (SD). Data were compared by 109 nonparametric analyses with Wilcoxon's matched pairs test, in which the median 110 was used to calculate significant differences. Spearman correlation coefficients 111 were calculated to investigate the correlation between measurements obtained 112 with the two anticoagulants. All statistical analyses were performed using the 
113 statistical software GraphPad Prism 5, version 5.04 (GraphPad Software, San 114 Diego, CA).

115 When measurements were below the lower limit of detection (LLD, $<5 \%$ of 116 measurements), we assigned a value equal to the midpoint between the LLD and 117 zero.

\section{Results and Discussion}

120 Significant differences in the measurements of all adipokines, except TNF- $\alpha$, were 121 observed in plasma samples collected in different anticoagulants (Fig. 1). IL-6, 122 leptin, adiponectin and CRP showed a significantly higher concentration in heparin 123 plasma compared to citrate.

124 However, despite these differences in absolute levels, the measurements of all 125 adipokines were highly correlated each other, except for TNF- $\alpha$ (Fig. 2). We can 126 speculate that, even though the measured levels differ, the same variation between

127 individuals can be detected, as demonstrated by the significant correlations.

128 TNF- $\alpha$ measurements differed from the other mediators both with regard to the 129 effect of anticoagulant and the lack of correlation between the levels in the paired 130 samples. These differences could be due to the very low levels, quite undetectable, 131 of this cytokine in our samples. To explain these results we performed also an 132 ELISA assay for TNF- $\alpha$ on the same samples: by this way we confirmed the absence 133 of significant differences in the levels of TNF- $\alpha$ in plasma samples obtained using 134 different anticoagulants. On the contrary, correlation analysis of ELISA results 135 obtained with the two anticoagulants was statistically significant $(\mathrm{r}=0.811 ; \mathrm{p}=$ 
136 0.004), confirming the results obtained with the other adipokines (data not 137 shown).

138 We carried out this study with explorative purposes only on ten subjects, with the 139 aim to understand if the cross-comparison of levels or profiles of adipokines 140 performed in different anticoagulants (e.g. results collected from various studies) 141 could be performed.

142 Adipokines are involved in the regulation of metabolism, insulin sensitivity, and 143 inflammation and serum concentrations of various adipokines are associated with 144 obesity, metabolic and cardiovascular diseases [1, 2]. It has been therefore 145 hypothesized that serum adipokine levels may serve as predictors of obesity146 related diseases or the individual disease outcomes [11].

147 Our study focused the attention on the measurement of five adipokines. 148 Anticoagulants effect on IL-6 and TNF- $\alpha$ measurements has been already described 149 in recent papers [3-6], while only a few of them described the effect on leptin [3,5] 150 or CRP [5], and none, to our knowledge, reported anticoagulants effect on 151 adiponectin measurement. However, the importance of such a study is underlined 152 by the proposal of using adiponectin as a clinical biomarker for several diseases 153 [12]. Lifestyle modification with visceral fat reduction combined with targeted 154 therapeutic interventions designed to improve adiponectinemia seem to be 155 potential clinically useful strategies to prevent obesity-related diseases, including 156 type 2 diabetes, cardiovascular diseases and malignancies [12].

157 Significant differences observed between different plasma samples in IL-6, leptin 158 and CRP levels were not in agreement with previous data reported by Biancotto et 159 al. [5], however, we measured analyte levels in fresh sample while they performed 
160 the assay on frozen specimens. When we did the same test on frozen samples,

161 statistically significant differences between plasma samples (LiEp vs. NaCitr) were

162 no more observed for IL-6 measurements but were still significant for leptin

163 measurements ( $\mathrm{p}=0.002$, Supplementary Materials Fig. S1). However, we observed

164 a significant difference between fresh and frozen plasma samples with Li Ep for IL-

1656 measurements $(\mathrm{p}<0.001$, Fig. S1) and between fresh and frozen plasma samples

166 both with LiEp and NaCitr for leptin measurements ( $p=0.002$, Fig. S1). Moreover,

167 these statistically significant results always showed a significant correlation (IL-6

168 LiEp fresh vs. frozen $\mathrm{r}=0.988, \mathrm{p}<0.0001$; Leptin LiEp fresh vs. frozen $\mathrm{r}=1$,

$169 \mathrm{p}<0.0001$; Leptin NaCitr fresh vs. frozen $\mathrm{r}=0.939, \mathrm{p}<0.002$; Supplementary

170 Materials Fig. S2). Previous papers on anticoagulant effect on cytokine

171 measurements often showed undetectable levels of IL-6, TNF- $\alpha$ or leptin, probably

172 because samples were stored at $-80^{\circ} \mathrm{C}$ for a different time and affected by multiple

173 freeze-thawing cycles $[3,5,6]$.

174 We dedicated our attention only at plasma samples, but differences between

175 plasma and serum cytokine measurements have been also described. However,

176 results are often discordant: for many analytes, high correlation were observed

177 between serum and plasma levels even when absolute levels differed. In fact, the

178 removal of fibrinogen, platelets and other circulating proteins from the plasma

179 during serum preparation could influence the presence or detection of an analyte.

180 Also during the cascade of coagulation, activation of cellular elements can release

181 inflammatory mediators, that may affect cytokine levels. This kind of information

182 are available for IL-6 and TNF- $\alpha$ [3-6], leptin [3, 5] or CRP [5], but are not available

183 for adiponectin. Possible differences between serum and plasma samples should 
184 be taken into consideration also when measuring adipokine levels and further 185 evaluations are needed.

186 We used Luminex multiplex bead-based technology, with the antibody kits 187 purchased from Bio-Rad or Merk (see Section 2). Previous studies compared the 188 performance of multiplex microbead assays between various vendors, and it was 189 noted that the consistency between the vendors was high only for some analytes $190[13,14]$. Though our conclusions are broadly applicable, specific care must be 191 taken when other kits or suppliers are used.

\section{Conclusions}

194 Our results emphasize, as already demonstrated for cytokines/chemokines [3-7], 195 the need to consider sample collection methods carefully when a study requires 196 measurement of adipokines in the peripheral blood. Cross comparison of levels or 197 profiles of adipokines performed in different anticoagulants should be avoided. 198 However, we observed a correlation between levels in plasma samples obtained 199 with different anticoagulants, and a possible solution might be the normalization 200 of values from one type of samples to an alternative type. Despite this possibility, 201 Jung and $\mathrm{Wu}$ demonstrated that this kind of conversion is complicated, and 202 calculations based on correlation analyses alone are not sufficient [15].

\section{Acknowledgements}

205 This work was supported by grants from the Associazione Italiana per la Ricerca 206 sul Cancro (project number IG13273, V.K.). Partial funding for this project has also 207 been received from the Compagnia di San Paolo (www.compagniadisanpaolo.it) 


\section{References}

212 [1] N. Ouchi, J.L. Parker, J.J. Lugus, K. Walsh, Adipokines in inflammation and 213 metabolic disease, Nat Rev Immunol, 11 (2011) 85-97.

214 [2] M. Bluher, C.S. Mantzoros, From leptin to other adipokines in health and 215 disease: facts and expectations at the beginning of the 21st century, Metabolism, $21664(2015) 131-145$.

217 [3] T.H. Tvedt, K.P. Rye, H. Reikvam, A.K. Brenner, O. Bruserud, The importance of 218 sample collection when using single cytokine levels and systemic cytokine profiles 219 as biomarkers--a comparative study of serum versus plasma samples, J Immunol 220 Methods, 418 (2015) 19-28.

221 [4] V.V. Krishnan, R. Ravindran, T. Wun, P.A. Luciw, I.H. Khan, K. Janatpour, 222 Multiplexed measurements of immunomodulator levels in peripheral blood of 223 healthy subjects: Effects of analytical variables based on anticoagulants, age, and 224 gender, Cytometry B Clin Cytom, 86 (2014) 426-435.

225 [5] A. Biancotto, X. Feng, M. Langweiler, N.S. Young, J.P. McCoy, Effect of 226 anticoagulants on multiplexed measurement of cytokine/chemokines in healthy 227 subjects, Cytokine, 60 (2012) 438-446.

228 [6] W. de Jager, K. Bourcier, G.T. Rijkers, B.J. Prakken, V. Seyfert-Margolis, 229 Prerequisites for cytokine measurements in clinical trials with multiplex 230 immunoassays, BMC Immunol, 10 (2009) 52.

231 [7] H.L. Wong, R.M. Pfeiffer, T.R. Fears, R. Vermeulen, S. Ji, C.S. Rabkin, 232 Reproducibility and correlations of multiplex cytokine levels in asymptomatic 233 persons, Cancer Epidemiol Biomarkers Prev, 17 (2008) 3450-3456.

234 [8] C. Belabani, S. Rajasekharan, V. Poupon, T. Johnson, A. Bar-Or, A condensed 235 performance-validation strategy for multiplex detection kits used in studies of 236 human clinical samples, J Immunol Methods, 387 (2013) 1-10.

237 [9] K. Skogstrand, Multiplex assays of inflammatory markers, a description of 238 methods and discussion of precautions - Our experience through the last ten years, 239 Methods, 56 (2012) 204-212.

240 [10] D.A. Vignali, Multiplexed particle-based flow cytometric assays, J Immunol 241 Methods, 243 (2000) 243-255.

242 [11] L.F. Van Gaal, I.L. Mertens, C.E. De Block, Mechanisms linking obesity with 243 cardiovascular disease, Nature, 444 (2006) 875-880.

244 [12] K. Kishida, T. Funahashi, I. Shimomura, Adiponectin as a routine clinical 245 biomarker, Best Pract Res Clin Endocrinol Metab, 28 (2014) 119-130.

246 [13] S.S. Khan, M.S. Smith, D. Reda, A.F. Suffredini, J.P. McCoy, Jr., Multiplex bead 247 array assays for detection of soluble cytokines: comparisons of sensitivity and 248 quantitative values among kits from multiple manufacturers, Cytometry B Clin 249 Cytom, 61 (2004) 35-39.

250 [14] A.M. Dupuy, N. Kuster, G. Lizard, K. Ragot, S. Lehmann, B. Gallix, J.P. Cristol, 251 Performance evaluation of human cytokines profiles obtained by various 
252 multiplexed-based technologies underlines a need for standardization, Clin Chem 253 Lab Med, 51 (2013) 1385-1393.

254 [15] K. Jung, C.Y. Wu, Methodological weakness in using correlation coefficients for 255 assessing the interchangeability of analyte data between samples collected under 256 different sampling conditions--the example of matrix metalloproteinase 9 257 determined in serum and plasma samples, Clin Chem Lab Med, 48 (2010) 733-736. 